\title{
Un agoniste peptidique hautement sélectif du récepteur de la morphine a été découvert dans le cerveau
}

\begin{abstract}
J. Zadina et al. (New Orleans, LO USA) viennent de rapporter la caractérisation d'un nouveau neuropeptide qui pourrait bien représenter l'équivalent endogène de la morphine [1]. Il y a plus de 20 ans, on a montré que les substances opiacées, originellement dérivées de l'opium, exercent leur activité analgésique puissante et leur action psychotrope euphorisante par l'intermédiaire de trois récepteurs classiquement nommés $\mu, \delta$ et $\mathrm{k}$ $\left(\mathrm{m} / \mathrm{s} n^{\circ} 10\right.$, vol. 8 , p. 1115) [2]. L'existence de ces récepteurs, abondamment exprimés à la surface de nos neurones, laissait supposer la présence de ligands endogènes capables d'agir sur ces récepteurs dans les conditions physiologiques. Les premiers opiacés endogènes isolés à partir de cerveau furent les enképhalines (1975), bientôt suivies de la $\beta$-endorphine et des dynorphines.
\end{abstract} Tous ces peptides possèdent une séquence amino-terminale identique (Tyr-Gly-Gly-Phe) et dérivent de trois précurseurs protéiques dont les gènes ont été clonés au début des années 1980. Le système opioïde dans son ensemble se composait donc, jusqu'à présent, de trois récepteurs et de trois familles de peptides. Son rôle dans la modulation de la réponse à la douleur et au stress, dans le contrôle des émotions, dans la régulation des fonctions autonomes, du système neuro-endocrinien et de la réponse immunitaire, est depuis largement démontré.

De l'étude des affinités respectives entre les différents protagonistes du système, ressortait la notion d'une sélectivité de reconnaissance relativement faible de la part de chaque famille de peptides vis-à-vis des trois sante qu'aucun de ces peptides ne semblait capable de cibler spécifiquement le récepteur $\mu$. Les enképhalines et les dynorphines présentent, respectivement, une sélectivité pour les récepteurs $\delta$ et $\kappa$. La $\beta$-endorphine se fixe avec une faible affinité sur le récepteur $\kappa$ et montre une bonne affinité à la fois pour les récepteurs $\delta$ et $\mu$. Ainsi le récepteur $\mu$, qui d'après de nombreuses données serait le médiateur majeur des effets autant bénéfiques qu'indésirables de la morphine [3], semble fonctionner de façon "non spécifique" dans la physiologie normale. La question était ouverte de savoir s'il restait encore à découvrir la vraie "morphine endogène ».

Les résultats de Zadina et al. indiquent que tel est le cas. Leur découverte est fondée sur l'isolement de peptides issus du cerveau au début des années 1990, dans leur propre laboratoire. L'un d'eux (Tyr-W-MIF-l ou Tyr-Pro-Trp-Gly-NH2) présentait des propriétés opiacées, avec une sélectivité raisonnable pour le récepteur $\mu$ par rapport aux deux autres récepteurs (deux ordres de grandeur) mais une affinité très modeste (70 nM). L'étude de peptides synthétiques dans lesquels la position $4 \mathrm{du}$ peptide fut remplacée par les 20 acides aminés naturels a permis d'obtenir un peptide synthétique de séquence Tyr-Pro-Trp-Phe présentant une affinité et une sélectivité remarquables pour le récepteur $\mu$. L’idée que ce peptide puisse réellement exister dans le système nerveux fut un pari on ne peut plus hasardeux ! C'est pourtant pour vérifier cette hypothèse que les auteurs produisirent un anticorps spécifique de ce peptide afin de tenter de détecter, puis de purifier le peptide à partir de cortex de bouf.

Ce pari fut gagné. Le peptide a bel et bien été isolé. La structure du peptide naturel est Tyr-Pro-Trp-Phe- $\mathrm{NH}_{2}$ (endomorphine-1). Il présente une forte affinité pour le récepteur $\mu$ (constante d'affinité inférieure au nanomolaire) et reconnaît ce récepteur respectivement 4000 et 15000 fois mieux que les récepteurs $\delta$ et $\kappa$. De plus, lorsqu'il est injecté in vivo chez la souris, il se comporte comme un analgésique d'une puissance comparable à celle de la morphine et montre une durée d'action bien supérieure à celle de tous les peptides opiacés endogènes connus à ce jour. Un deuxième peptide apparenté, de séquence Tyr-Pro-Phe-Phe$\mathrm{NH}_{2}$ (endomorphine-2), a été coisolé avec le premier et présente des propriétés similaires. Les deux endomorphines seraient moins abondantes que les enképhalines mais pourraient exister en quantités similaires à celles de la $\beta$-endorphine ou de la dynorphine dans le cerveau. Il reste à démontrer que ce peptide endogène existe réellement en clonant le gène responsable de sa synthèse. On peut anticiper dès à présent que ce gène pourrait coder pour un grand précurseur polypeptidique à partir duquel les endomorphines seraient engendrées par protéolyse, comme c'est le cas d'autres précurseurs de neuropeptides. L'analyse de la structure primaire de ce précurseur pourrait alors mener à la découverte d'autres peptides biologiquement actifs.

L'étude détaillée de l'activité biologique des endomorphines reste à faire. Leur activité analgésique s'accompagne-t-elle des effets secon- 
daires connus de la morphine (dépression respiratoire, nausées, tolérance et dépendance) ? C'est probable dans la mesure où, quelles que soient les approches utilisées jusqu'à présent, on n'a pu dissocier l'ensemble de ces effets qui sont corelayés par le récepteur $\mu$ [3]. Pourtant, on ne peut exclure que les endomorphines, dont la structure est extrêmement différente de celle des alcaloïdes, agissent différement sur la protéine réceptrice et produisent des effets biologiques distincts de ceux des dérivés de l'opium.

Enfin, après l'explosion dans la découverte des peptides de type enképhaline qui a caractérisé la fin des années 1970 et les deux décennies d'accalmie qui ont suivi - accompagnées de cette impression d'avoir fait le tour d'une question -, on peut se demander si la découverte des endomorphines marque un soubresaut dans l'histoire des opiacés endogènes ou si l'on assiste au début d'une nouvelle ère au cours de laquelle on découvrira d'autres ligands hautement sélectifs.

B.K.

1. Zadina EJ, Hackler L, Ge LJ, Kastin AJ. A potent and selective endogenous agonist for the $\mu$-opiate receptor. Nature $1997 ; 386: 499$ 502.

2. Noel F, lourgenko V, Pouille Y, Hanoune J. I.es mécanismes d'action moléculaire des opiacés. Med Sci $1994 ; 10: 1116-26$.

3. Kieffer B, Matthes H, Maldonado R. Mécanisme d'action de la morphine. Med Sci $1997 ; 13$ : 232-5.

\section{BRÈVES}

Les canaux édités. médecine/sciences a maintes fois fait allusion aux énigmatiques phénomènes de "corrections sur épreuves", désignés en anglais par le terme editing: En réalité, ce mot regroupe une grande diversité de phénomènes, qui vont du changement considérable de la séquence des transcrits primitifs des ARN mitochondriaux de trypanosomes, avec additions ou délétions multiples de résidus uridine $(\mathrm{m} / \mathrm{s}$ $n^{\circ} 11$, vol. $11, p .1622$ ), à la désamination sélective de résidus cytosine (dans le transcrit de l'apolipoprotéine B) $\left(\mathrm{m} / \mathrm{s} n^{\circ} 10\right.$, vol. $\left.5, p .787\right)$ ou adénosine [1]. C'est la désamination de l'adénosine en inosine, qui est lue comme une guanosine, qui en cause dans l'édition des transcrits codant pour des chaînes des récepteurs du glutamate de type AMPA et kainate $\left(\mathrm{m} / \mathrm{s} n^{\circ} 10\right.$, vol. 7 , p. 1098). Dans ces récepteurs, le changement du codon CAG (glutamine, Q) en CGG (arginine, R) est le plus fréquent (sites $R / G$ des transcrits codant pour les chaînes Glu-R-B, Glu-R-C et Glu-R-D). Au niveau de la sous-unité Glu-R-6, on trouve des changements isoleucine $\rightarrow$ valine et tyrosine cytosine. Ces remplacements d'acides aminés provoqués par le phénomène d'édition modifient considérablement la perméabilité des canaux et ont une importance fonctionnelle vitale: des souris transgéniques incompétentes pour l'édition des ARN codant pour la chaîne Glu-R-B ont un syndrome épileptique rapidement mortel $(\mathrm{m} / \mathrm{s}$ $n^{\circ} 2$, vol. 12, p. 262). Le mécanisme de la sélection des sites édités est maintenant connu: des séquences exoniques et introniques partiellement complémentaires entraînent la formation de portions d'ARN double brin qui sont reconnues par des adénosines désaminases dont plusieurs espèces moléculaires ont maintenant été identifiées : l'enzyme dsRAD/DRADA (doublestranded RNA adenosine deaminase) édite les sites $\mathrm{R} / \mathrm{G}$ du transcrit Glu$\mathrm{R}-\mathrm{B}$ et le site $\mathrm{Q} / \mathrm{R}$ des transcrits GluR-5 et Glu-R-6. Deux autres enzymes ont été récemment identifiées: RED1, de spécificité proche de celle de dsRAD/DRADA, et RED2 dont l'activité n'a pas encore été étudiée. Burns et al. (Nashville, TX, USA) démontrent maintenant que ce phénomène d'édition peut également être observé au niveau du transcrit codant pour un récepteur de la sérotonine, le récepteur $5-\mathrm{HT}_{2 \mathrm{c}}$ Cette édition intéresse trois sites contigus $\mathrm{I} / \mathrm{V}, \mathrm{N} / \mathrm{S}, \mathrm{l} / \mathrm{V}$ (isoleucine $\rightarrow$ valine et asparagine $\rightarrow$ sérine) situés au niveau de la seconde boucle intracellulaire du récepteur à sept passages transmembranaires [2]. Cette région intervient dans le couplage avec les protéines $\mathrm{G}$, et donc dans la transmission du signal sérotoninergique (baisse de l'activation de l'enzyme effecteur, la phospholipase C). Le récepteur modifié par édition est partiellement inactif. Dans les différentes régions du cerveau, co-existent des formes natives, les plus actives, et des formes éditées, moins actives. La proportion de formes natives et éditées varie cependant suivant les régions, si bien que l'efficacité de la transmission du signal sérotoninergique peut être modulée par ce phénomène d'édition différentielle. L'une des fonctions du récepteur $5-\mathrm{HT}_{2 c}$ est de contrôler l'appétit: les agonistes de ce récepteur sont anorexigènes alors que les souris dont les deux allèles du gène 5-HT $T_{2 C}$ ont été invalidés sont hyperphagiques et obèses $\left(\mathrm{m} / \mathrm{s} n^{\circ} 7\right.$, vol. 11, p. 1053). L'édition du récepteur $\mathrm{HT}_{2 \mathrm{c}}$ pourrait donc jouer un rôle dans le contrôle central de la satiété.

[1. Auxilien S, Grosjean H. Med Sci 1995 ; 11 : 1089-98.]

[2. Burns CM, el al. Nature 1997; 387 : 303-8.]

\section{JOURNÉE JEAN-CLAUDE DREYFUS DE GÉNÉTIQUE ET DE PATHOLOGIE MOLÉCULAIRES RECEPTEURS et MALADIES RECEPTORS and DISEASES \\ Vendredi 19 septembre 1997, Renseignements et inscriptions, voir page 2 de couverture}


Invalidation de l'utrophine: impact bien décevant à la jonction neuromusculaire. L'utrophine est une protéine quasi ubiquiste mais dont la localisation dans le muscle est restreinte, chez l'adulte, à la jonction neuromusculaire. Son homologie avec la dystrophine est telle qu'elle est capable de compenser le déficit de cette dernière dans le muscle de souris mutantes $m d x$ ( $m / s n^{\circ} 2$, vol. 13, p. 256). Différentes publications antérieures avaient suggéré que l'utrophine jouerait un rôle important dans la formation ou le maintien des synapses; en effet, en culture cellulaire, les agrégations des récepteurs de l'acétylcholine au niveau de la membrane musculaire, caractéristiques de la membrane postsynaptique, sont associées à une accumulation d'utrophine; de plus, les animaux dépourvus de protéines impliquées dans la jonction synaptique comme les mutants rapsyne-ou agrine $^{-/-}$perdent cette accumulation de l'utrophine. Du fait de la très grande taille du gène qui, $a$ priori, devrait en faire une cible des mutations, certains auteurs avaient même fait le pari que si aucune mutation du gène de l'utrophine n'avait encore été dépistée chez l'homme, c'est que celles-ci devraient être létales. Toutes ces supputations semblent démenties par deux récents articles publiés conjointement dans le Journal of Cell Biology relatant l'invalidation du gène de l'utrophine. L'équipe anglaise [1] a introduit un gène traceur à l'extrémité amino-terminale du gène, laissant potentiellement exprimées les isoformes les plus distales, alors que l'équipe américaine [2] a muté son extrémité carboxyterminale invalidant ainsi toutes les isoformes connues à ce jour. Les résultats observés sont néanmoins identiques : les animaux sont $a$ priori normaux et fertiles, aucune anomalie histologique des poumons, du rein, du cerveau, du cœur ou des muscles, principaux tissus d'expression de l'utrophine, n'est notée et la structure générale de la jonction neuromusculaire paraît normale.
On note notamment une innervation des muscles par un seul axone moteur, l'absence d'innervation extrasynaptique, habituellement rencontrée dans les autres invalidations de gènes de la synapse comme l'agrine ou la rapsyne, une distribution normale des récepteurs de l'acétylcholine. Une étude plus fine de la jonction neuromusculaire fait néanmoins apparaître des anomalies discrètes des repliements de la membrane postsynaptique qui semblent moins nombreux que chez la souris normale avec une variabilité en fonction des muscles étudiés - notamment détectée dans les muscles intercostaux et le soléaire qui se traduit par une diminution mineure de la densité des récepteurs de l'acétylcholine de l'ensemble des synapses. Par ailleurs, les autres protéines comme toutes les protéines du complexe associé à la dystrophine, la laminine, la rapsyne, l'agrine, le récepteur MusK, sont normalement synthétisées. L'implication de l'utrophine dans la synaptogenèse paraît donc assez limitée. Il faudra attendre l'invalidation des autres gènes de la famille dystrophine, comme la dystrobrévine (protéine qui présente une homologie de $37 \%$ avec le domaine carboxy-terminal de la dystrophine et qui est également concentrée à la jonction neuromusculaire), ou la DRP2 (protéine essentiellement cérébrale qui présente une organisation très ressemblante aux domaines riches en cystéine et au domaine carboxy-terminal de l'utrophine) et les croisements de ces différents mutants avant de pouvoir incriminer une désormais classique redondance fonctionnelle et surtout espérer comprendre la fonction précise de toutes ces nouvelles protéines.

[1. Deconinck AE, et al. J Cell Biol 1997 ; 136 : 883-94.]

[2. Grady RM, et al. J Cell Biol 1997; 136: 871-82.]
- Préséniline, maladie d'Alzheimer et signal Notch. La préséniline 1 , en cause dans une forme familiale de maladie d'Alzheimer d'apparition précoce $\left(\mathrm{m} / \mathrm{s} n^{\circ} 11\right.$, vol. 11, p. 1610) semble être l'homologue chez les mammifères de la protéine SEL-12 de Caenorhabditis elegans. Les souris déficientes en préséniline $1\left(P_{s} 1^{-1-}\right)$ meurent à la naissance avec de nombreuses anomalies du développement intéressant le squelette axial et les ganglions spinaux. Ce phénotype suggère que la segmentation et la différenciation des somites est anormale. P.C. Wong et al. (Baltimore, MD; Rahway, NJ, USA et Essex, GB) montrent que, chez les embryons déficients en préséniline 1, l'expression des gènes Notch1 et Dll1 (delta-like gene 1) est très réduite au niveau du mésoderme para-axial. La protéine Dll1 est un ligand du récepteur Notch [1]. Par ailleurs, Baumeister et al. (Munich, Allemagne) ont étudié l'équivalence fonctionnelle entre la préséniline 1 et la protéine SEL-12 de Caenorhabditis elegans [2]. Ils ont en effet introduit chez un mutant de ce nématode, soit le gène $P S-1$ normal, soit un gène $P S-1$ muté, isolé d'un malade souffrant d'une forme familiale d'Alzheimer. L'anomalie de la ponte des œufs caractérisant les mutants sel-12 est totalement corrigée par le gène $P S$ 1 normal mais non par le gène $P S-1$ muté. Par conséquent, la préséniline 1 est un homologue fonctionnel vrai de SEL-12 chez Caenorhabditis elegans, et est indispensable, chez le ver comme chez les mammifères, à l'action normale du gène Notch. Précédemment, il a été montré que les mutations des gènes codant pour les présénilines 1 et 2 étaient associées à une accumulation du peptide $\beta$-amyloïde, suggérant que les présénilines pouvaient être impliquées dans le routage intracellulaire des protéines $\left(m / s n^{\circ} 1\right.$, vol. 13 , p. 106). On ne sait pas à ce jour s'il existe une relation entre cette action supposée des présénilines et leur rôle démontré dans la transmis- 


\section{BRÈVES}

sion du signal Notch au cours du développement.

[1. Wong PC, et al. Nature 1997; 387: 288-92. ]

[2. Baumeister R, et al. Genes Funct 1997; 1 : 149-59.]

Cstrogènes et maladie d'Alzheimer. Depuis plusieurs décennies, on sait que les œstrogènes agissent au niveau du SNC: ils influent sur les états affectifs et interviennent dans le contrôle des fonctions sensori-motrices en modifiant des neurotransmissions catécholaminergiques. Récemment, des études biochimiques et cliniques ont montré que les œstrogènes étaient également capables d'améliorer les fonctions cognitives et, en particulier, de retarder l'apparition des symptômes de la maladie d'Alzheimer chez les femmes [1]. Confirmant les travaux pionniers de Fillit dans les années 1980, l'équipe de R. Mayeux (New York, NY, USA) [1] vient en effet de publier à ce sujet une étude épidémiologique réalisée sur 1124 femmes ménopausées d'environ 75 ans (en bonne santé au début de l'étude). Les résultats semblent montrer clairement que le déclenchement de la maladie d'Alzheimer est retardé de manière significative (de plusieurs années en moyenne) chez les femmes traitées par les œstrogènes et que, d'autre part, le risque relatif d'apparition des symptômes est réduit chez ces patientes $(5,8 \%$ parmi les femmes traitées par les œstrogènes contre $16 \%$ chez les non-traitées). La durée du traitement œstrogénique semble également jouer un rôle important. Quelles sont les bases biochimiques susceptibles d'expliquer ce phénomène? Luine et McEwen (New York, NY, USA) avaient observé que les œstrogènes augmentaient les taux de choline acétyltranférase (ChAT) des neurones du cerveau antérieur projetant vers l'hippocampe et le cortex cérébral (zones du cerveau particu- lièrement importantes dans la mémorisation et l'apprentissage) (pour revue, voir [2]). D'autre part, une perte massive des neurones cholinergiques dans les noyaux du cerveau antérieur a été décrite chez les malades atteints de maladie d'Alzheimer. Les œstrogènes pourraient ainsi restaurer une partie de la transmission cholinergique défectueuse. Les œstrogènes semblent également capables d'augmenter le nombre de contacts synaptiques dans l'hippocampe, ainsi que la quantité de récepteurs glutamatergiques de type NMDA (N-méthyl-Daspartate) impliqués dans la potentialisation synaptique à long terme (LTP) $\left(m / s n^{\circ} 8\right.$, vol. 8, p. 870). Ces données pourraient constituer un support biochimique à l'amélioration des fonctions cognitives par le stéroïde. Une des théories actuelles concernant le mode d'action des œstrogènes sur la régénération neuritique serait une coopération entre l'hormone et la voie de transmission du signal d'une des principales neurotrophines, le NGF (nerve growth factor, facteur de croissance du nerf). Cette théorie met en jeu le récepteur nucléaire des œstrogènes et l'activation de gènes spécifiques (tels le récepteur du NGF ou l'enzyme ChAT) [2]. Très récemment, Behl et al. (Munich, Allemagne) [3] ont montré un effet neuroprotecteur direct des œestrogènes sur des neurones d'hippocampe vis-à-vis de la toxicité de la protéine $\beta$-amyloïde (s'accumulant dans les plaques séniles des patients atteints de la maladie d'Alzheimer) et des acides aminés excitateurs tels le glutamate. Il s'agirait d'une activité anti-oxydante du stéroïde, se traduisant par une diminution de l'accumulation de peroxydes intracellulaires impliqués dans la dégénérescence neuronale. Les effets protecteurs des œstrogènes vis-à-vis du stress oxydatif sont partagés par d'autres dérivés stéroïdiens possédant un groupement hydroxyle dans une position définie d'un des anneaux de la molécule et n'impliquent pas l'activation du récepteur nucléaire des œstrogènes.
Cette découverte semble de première importance pour la conception de nouvelles molécules stéroïdiennes à visée thérapeutique (dépourvues des effets secondaires des oestrogènes, notamment chez les hommes) dans la maladie d'Alzheimer ou d'autres maladies neurodégénératives liées au stress oxydatif.

HIPl, un sérieux partenaire de la huntingtine. La maladie de Huntington fait partie des affections liées à la synthèse de protéines possédant des répétitions allongées de résidus glutamine, conséquence d'une extension de triplets CAG [1, 2]. La maladie est dominante, et les symptômes créés chez la souris par une mutation homozygote du gène en cause, codant pour la huntingtine, n'entraîne pas les signes neurologiques caractéristiques de la chorée de Huntington $\left(m / s n^{\circ} 6 / 7\right.$, vol. 12, p. 804). Cependant, la seule présence d'une séquence protéique polyglutamine dans les cellules nerveuses n'explique pas aisément la symptomatologie car les signes cliniques de plusieurs maladies associées au même type de mutation sont très différents. C'est ainsi que, quoique la huntingtine y soit synthétisée abondamment, les cellules de Purkinje sont épargnées dans la chorée de Huntington alors qu'elles sont détruites progressivement dans l'ataxie spinocérébelleuse de type 1 , une maladie dans laquelle la protéine ataxine contient également une répétition allongée de résidus glutamine $\left(\mathrm{m} / \mathrm{s} n^{\circ} 4\right.$, vol. $\left.10, p .472\right)$. Ces données suggèrent que le gain de fonction associé à la séquence polyglutamine est néanmoins spécifique des protéines au niveau desquelles se rencontrent ces répétitions. Ces résultats ont entraîné une recherche fébrile de partenaires privilégiés des protéines modifiées dans ces maladies, notamment de la huntingtine, dont l'interaction avec les protéines mutées pourrait être anormale, augmentée ou diminuée 


\section{BRÈVES}

(m/s $n^{\circ} 4$, vol. 12, p. 535). Deux équipes rapportent maintenant le clonage d'un $\mathrm{ADN}$ complémentaire isolé par la technique du doublehybride dans la levure et codant pour la même protéine, HIPl (huntingtin interacting protein 1) [3, 4]. Ce partenaire semble un candidat sérieux pour expliquer certaines des anomalies associées à la chorée de Huntington. En effet, HIPl présente de fortes similitudes avec le produit du gène SLA2 de levure. Ce gène code pour une protéine qui semble essentielle à l'assemblage et à la fonction du cytosquelette sousmembranaire. De plus, la protéine HIPl est synthétisée dans le système nerveux central dans les mêmes neurones que la huntingtine avec laquelle elle est co-localisée au niveau de la membrane. L'interaction entre la huntingtine et HIPl est diminuée progressivement alors que s'allonge la répétition de glutamines. Ces résultats suggèrent qu'une perte de l'interaction entre la huntingtine mutée et HIPl pourrait perturber le cytosquelette des neurones et ainsi induire leur mort prématurée. Cependant, cette hypothèse n'explique pas aisément l'absence de stigmates de chorée de Huntington chez des souris déficientes en huntingtine, animaux chez lesquels, évidemment, il ne peut y avoir d'interaction avec HIPl. Par conséquent, si HIPl est bien en cause dans la symptomatologie de la chorée de Huntington, le mécanisme pourrait en être plus l'accumulation de huntingtine mutée n'interagissant pas avec HIPl, que la présence en ellemême de cette protéine HIPl non complexée à l'huntingtine. HIP1 s'ajoute donc à la liste des partenaires de la huntingtine qui ont été proposés pour jouer un rôle dans la symptomatologie de la chorée de Huntington, après la glyceraldéhyde 3-phospate déhydrogénase.. et d'autres $\left(\mathrm{m} / \mathrm{s} n^{\circ} 4\right.$, vol. $12, p .535$; $n^{\circ} 6 / 7$, vol. 12, p. 852). Cependant, la nature de la protéine HIP1 et sa co-localisation, cellulaire et sub-cellulaire, avec la huntingtine en font effectivement un candidat particulièrement sérieux au rôle de coupable potentiel de la mort neuronale observée chez les malades.

[1. Mandel JL. Med Sci 1996; 12 (suppl n 10): 100-8.]

[2. Néri C, et al. Med Sci 1996; 12 1361-9.]

[3. Wanker E, et al. Hum Mol Genet $1997 ; 6$ : 487-95.]

[4. Kalchman MA, et al. Nature Genet 1997 ; 16 : 44-53.]
ASSOCIATION POUR

\section{L'ÉTUDE DE LA PATHOLOGIE PÉDIATRIQUE}

(Association déclarée par la loi du Ier Juillet 1901 - JO 21 mai 1970) PRIX 60000 F

- L'Association pour l'Étude de la Pathologie Pédiatrique institue un prix annuel de soixante mille francs destiné à honorer un ravail scientifique de pathologie pédiatrique. Ce travail devra être original, publié ou à publier, et concerner explicitement les causes ou les mécanismes des maladies de l'enfant, ou apporter des progrès substantiels à leurs méthodes d'investigation.

- Ce prix s'adresse préférentiellement à des pédiatres, pathologistes ou chercheurs âgés de moins de quarante ans.

- Le lauréat recevra son prix lors d'une des réunions annuelles de la Division Française de l'Académie Internationale de Pathologie.

Les demandes d'informations et les dossiers de candidatures (en 8 exemplaires) doivent êrre adressées à :



Date limite de dépôt : le $\left.\right|^{\text {er }}$ novembre 1997

\section{GÉNÉTIQUE DE LA FERTILITÉ MASCULINE COLLIOURE, FRANCE 4-6 septembre 1997}

\section{Jeudi 4 septembre}

\section{GENETIC CONTROL OF SPERMA-} TOGENESIS

Genetic of sexual differenciation: C. Sultan (F)

Genetic control of spermatogenesis: N. Hecht (USA)

Mapping of the Y : D. Page (USA)

Epidemiology of oligo and azoospermia : A. Spira (F)

Genetic aspects of flagellar dyskinesia, globozoospermia: C. Gagnon (C) Urogenital dysgenesis and male infertility : P.N. Schlegel (USA)

Karyotype and oligospermia: A. Chandley (UK)

Heritability of sterility: $H$. Tournaye (B)

\section{Vendredi 5 septembre}

GENETIC OF THE SPERMATOZOA DNA packaging: $S$. Ward (USA)

Detection and characterization of chromosome abnormalities: R. H. Martin (C $X / Y$ separation : J.D. Schulman (USA) Oxydative damages of chromatin: D.Sakkas (S)

\section{SESSION DE POSTERS}

\section{TABLE RONDE ÉTHIQUE}

McDonough (USA), M. Serres (F), F. Collins (USA), A. Kahn (F)

\section{Samedi 6 septembre}

ROLE OF SPERMATOZOA IN EMBRYOGENESIS

Paternal effects on early embryogenesis : L. Janny (F)

Paternal inheritance of the centrosome G. Schatten (USA)

Gene imprinting : N. De Groot (IL)

Mitochondrial DNA : J. Cummins (Aus)

\section{Renseignements :}

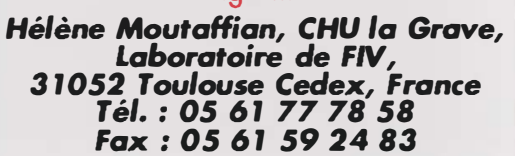

